techniques and can be used to assess pulmonary congestion and volume status at the bedside.

Aims To describe the reproducibility of lung POCUS when performed by an experienced echocardiographer, and the accuracy of assessments performed by novice practitioners. To explore the clinical usefulness of POCUS in the management of hospitalized patients with HF, who are judged as clinically euvolemic following inpatient therapy and for whom discharge is under consideration.

Method We performed POCUS of the lungs at the bedside for sequential inpatients with HF. The index exam was performed on the day in which the patient was judged clinically to be euvolemic. Based on this exam patients were separated into residual congestion (RC) and true euvolemic (TEu) groups. Each patient had at least 1 POCUS exam, RC group patients had further studies during their stay. The POCUS exam consisted of: imaging the lung bases for pleural fluid, 8 lung zones anteriorly for B-lines, and established IVC indices. All exams were done first by a single experienced echocardiographer. To obtain a measure of reproducibility, 120 stored loops were analysed blindly and independently by each author. We collected baseline demographic clinical and laboratory data, time to discharge from index POCUS, and readmission rates for all patients. For those in the RC group we report further in-hospital weight loss, and any adverse events occurring from further treatment.

Results Intraobserver reproducibility for assessment of pleural fluid and B-lines were 100\% and 98\% respectively. Novice practitioners reported presence/absence of pleural fluid and B-lines with an accuracy of $97 \%$ and $96 \%$ respectively. 17 sequential patients were recruited over 6 weeks. 9 patients were judged to have RC at the time of POCUS. These 9 form the RC group, the remaining 8 form the TEu group. Median age of the total group was 79 (IQR 70-83), 10/19 had HF with reduced EF, median peak NT-proBNP taken during the index admission was 4399 (IQR 3162-6575).
Corresponding figures for groups were RC: age 75 (IQR 7179), 5/9 HFrEF, and peak NT-proBNP 5530 (IQR $3653-$ 8300); TEu age 81 (IQR 68-83), 4/8 HFrEF, and peak NTproBNP 4010 (IQR 1671-4399). Median additional LOS for the RC group was 4 days (IQR 3-7). 7/8 TEu patients were discharged within 1 day of the index POCUS confirming euvolemia. No patient in either group was readmitted at 30 days. RC group patients had additional therapy under POCUS guidance with the aim of eliminating residual pulmonary congestion. An additional median weight loss of $4.8 \mathrm{Kg}$ (IQR $3.4-6.5 \mathrm{Kg}$ ) was achieved. $1 \mathrm{RC}$ group patient had an episode of hypotension requiring fluid replacement. 2 had residual pulmonary congestion persistent on discharge. No patient had a rise of serum creatinine greater than $50 \%$ from baseline.

Conclusion In this pilot study we show that RC is common in hospitalized patients with HF who are judged clinically to be euvolemic. We show that POCUS can provide a reproducible and clinically relevant assessment of RC and that this information can be used to guide further management.

\section{A QUALITATIVE ANALYSIS OF CARDIOLOGY PRACTITIONER OPINION REGARDING THE BENEFITS, ADVERSE EFFECTS AND CHALLENGES ASSOCIATED WITH THE POTENTIAL INTRODUCTION OF PUBLIC REPORTING OF PHYSICIAN EVENTS AND RESULTS (PROPER) TO IRELAND}

N Fitzpatrick. Cork University Hospital, Cork, Ireland

\subsection{6/heartjnl-2020-ICS.33}

Introduction The premise behind the public reporting of physician events and results (PROPER) is at first glance straight forward. By periodically publicly reporting

Abstract 33 Table 1 Likert Scale Questions used regarding PROPER

Q8. Public reporting at a physician level can lead to
improved patient outcomes.
Q9. Public reporting at an institution level can lead to
improved patient outcomes.
Q10. Public reporting of physician level outcomes
would lead to improved care for patients in Ireland
Q11. Public reporting of institution level outcomes
would lead to improved care for patients in Ireland.
Q12. Public reporting would significantly affect
referral patterns by GPs and other healthcare
providers if introduced in Ireland.
Q13. Public reporting would significantly influence
how patients choose their cardiologist if introduced
in Ireland

Q15. Risk adjusted mortality statistics provide a valid quality metric when assessing a cardiologists practice.

Q16. The introduction of public reporting would affect physician's willingness to intervene on high risk or critically ill patients who are at high risk of an adverse outcome.

Q17. The introduction of public reporting would affect your willingness to intervene on high risk or critically ill patients.

Q18. Risk adjustment tools exist to sufficiently mitigate against potential adverse behaviour of "cherry picking" of patients or avoidance of high risk patients.

Q19. Risk adjustment tools would likely lead to "gaming" or "up coding" behaviour by physicians or institutions to enhance their outcome reports.

Q20. Current information technology infrastructure and systems as typically found within healthcare in Ireland are adequate to capture data needed to measure outcomes and risk adjust patients. 
physicians' outcomes and events, patients are empowered to make more informed decisions when choosing their doctor. Such a registry helps to redress the information asymmetry found at the core of the decision-making problem for patients when attempting to choose their doctor. Examples of PROPER registries include the NICOR Adult PCI Registry in the UK which reports Percutaneous Coronary Intervention (PCI) outcomes for all operators in the UK. Another example (since 1991) is the New York State Cardiac Registry which publishes risk adjusted outcome statistics for all cardiothoracic surgeons operating in the State of New York. Currently, there are no PROPER registries in Ireland. There has only been publication of institution level outcomes through the National Healthcare Quality Reporting System (NHQRS). We surveyed practicing cardiologists and trainees in Ireland, the aim was to examine their perception of the benefits and potential harms associated with PROPER registries. The potential impact such registries might have on referral patterns, market share, how patients choose their cardiologist and whether risk adjustment algorithms are sufficient to protect against adverse behaviours.

Methods Data was collected by means of a 20-question survey circulated amongst attendees at the Irish Cardiac Society (ICS) Meeting 2019. An electronic copy of the survey was also distributed to cardiology practitioners and trainees known to the author but not encountered at the ICS conference. The questions (see table 1) were framed as statements, respondents were then asked to quantify their agreement or disagreement with the statement based on a 5 -point Likert scale.

Results A total of 39 responses to the survey were received (estimate $26 \%$ of all possible responses). Irish cardiologists are in general optimistic regarding the potential benefits of PROPER. Most markedly at an institution level, 81\% agreeing that it would improve patient care. $87 \%$ of respondents felt that referrals would be significantly affected by public reporting. $77 \%$ felt PROPER would impact market share of public versus private practice. $60 \%$ of responders considered mortality to be an appropriate quality assurance metric for cardiology (in contrast to only $15 \%$ found in similar studies). An overwhelming 97\% of respondents felt that public reporting would affect doctors' willingness to intervene on high risk patients. $87 \%$ of respondents felt that PROPER would lead to other adverse behaviours such as 'upcoding' or 'gaming.'

Conclusion In conclusion we see that Irish cardiologists in general hold an optimistic view regarding the potential benefits of PROPER. However, they are also aware of its potential for adverse unintended consequences. Responses to this survey were broadly in keeping with those founds in other studies performed in jurisdictions with active PROPER registries, though there were some notable differences.

\section{USE OF ULTRASOUND DERIVED CAROTID INTIMA- MEDIA THICKNESS AND PLAQUE VOLUME TO PREDICT SINGLE OR MULTIVESSEL CORONARY ARTERY DISEASE}

${ }^{1} \mathrm{~K}$ Owen, ${ }^{1}$ I Menown, ${ }^{2} \mathrm{~J}$ McLaughlin. ${ }^{1}$ Craigavon Area Hospital, Craigavaon, UK; ${ }^{2}$ Ulster University, UK

10.1136/heartjnl-2020-ICS.34
Background Increased carotid intima-media thickness (cIMT) has been extensively evaluated as a marker of cardiovascular (CV) risk. However, there is only limited evidence correlating cIMT measurements with anatomical severity of vascular disease. This study compared the value cIMT \pm other carotid measurements to predict the presence and severity of significant coronary artery disease (CAD).

Methods Patients were included with a history of ischaemictype chest pain or angina equivalent and undergoing either Invasive Coronary Angiogram (ICA) or Computed Tomography Coronary Angiography (CTCA). All patients underwent high-resolution B-mode ultrasound imaging to measure cIMT and B-Mode 3D-imaging to measure Total Plaque Volume (TPV) and Maximum Area Reduction (MAR) using latest generation dedicated hardware and software. cIMT normal ranges were defined by previous independent population studies. An operator, blinded to carotid measurements, defined the presence and severity CAD on ICA or CTCA. The study was supported by a European Union INTERREG VA Programme grant.

Results The study population comprised of 90 subjects $(73 \%$ male). Mean age was $66.2 \pm 11.63 \mathrm{SD}$ years (range, 42-88 years). CV risks factors included family history of CAD (74\%), current or ex-smoker (69\%), hypertension (66\%), a history of hyperlipidaemia (61\%) and diabetes (21\%). On ICA or CTCA, $62 \%$ of patients had severe disease (defined as at least $70 \%$ area stenosis or pressure wire positive) in at least one coronary artery, and 39\% had severe multivessel disease (in 2 or more vessels). The presence of elevated cIMT $\geq 50$ th percentile predicted the likelihood of severe multivessel CAD (relative risk [RR] $1.49 ; 47 \%$ vs $21 \%$; $\mathrm{p}=0.022)$ and the likelihood of severe CAD in at least one vessel (RR $1.65 ; 71 \%$ vs $43 \% ; \mathrm{p}=0.011$ ). Use of cIMT $\geq 75$ th percentile cut off did not increase predictive value. On 3D analysis, TPV in the top tertile predicted likelihood of severe CAD in at least one vessel (RR 1.4; 77\% vs 55\%; $\mathrm{p}=0.046)$. MAR did not add further to the predictive value. (Table 1).

\begin{tabular}{|c|c|c|c|c|c|c|}
\hline & $\begin{array}{l}\text { CIMT } \\
\geq 50 \text { th } \\
\text { percentile } \\
(n=62)\end{array}$ & $\begin{array}{l}\text { CIMT } \\
<50 \text { th } \\
\text { percentile } \\
(n=28)\end{array}$ & $\begin{array}{l}\mathrm{p} \\
\text { value }\end{array}$ & $\begin{array}{l}\text { cIMT } \\
\geq 75 \text { th } \\
\text { percentile } \\
(\mathrm{n}=41)\end{array}$ & $\begin{array}{l}\text { clMT } \\
<75 \text { th } \\
\text { percentile } \\
(n=49)\end{array}$ & $\begin{array}{l}p \\
\text { value }\end{array}$ \\
\hline $\begin{array}{l}\text { Severe CAD } \\
\text { in at least } \\
\text { one vessel }\end{array}$ & 44 & 12 & 0.011 & 31 & 25 & 0.016 \\
\hline $\begin{array}{l}\text { Severe } \\
\text { multivessel } \\
\text { CAD }\end{array}$ & 29 & 6 & 0.022 & 19 & 16 & 0.18 \\
\hline
\end{tabular}

Conclusions In a population of patients with ischaemic type chest pain or angina equivalent symptoms, elevated cIMT $(\geq 50$ th percentile) predicted an increased risk of severe CAD in at least one vessel and over double the risk of severe multivessel CAD. CIMT may thus be a useful tool to help triage patients most likely to benefit from invasive coronary investigation. 